\title{
Immunomics: a 21 st century approach to vaccine development for complex pathogens
}

\author{
KARINA P. DE SOUSA ${ }^{1,2}$ and DENISE L. DOOLAN ${ }^{1,2} *$ \\ ${ }^{1}$ Infectious Diseases Programme, QIMR Berghofer Medical Research Institute, Herston, QLD 4029, Australia \\ ${ }^{2}$ School of Medicine, University of Queensland, St. Lucia, QLD 4072, Australia
}

(Received 24 May 2015; revised 3 fuly 2015; accepted 25 fuly 2015; first published online 7 Fanuary 2016)

SUMMARY

Immunomics is a relatively new field of research which integrates the disciplines of immunology, genomics, proteomics, transcriptomics and bioinformatics to characterize the host-pathogen interface. Herein, we discuss how rapid advances in molecular immunology, sophisticated tools and molecular databases are facilitating in-depth exploration of the immunome. In our opinion, an immunomics-based approach presides over traditional antigen and vaccine discovery methods that have proved ineffective for highly complex pathogens such as the causative agents of malaria, tuberculosis and schistosomiasis that have evolved genetic and immunological host-parasite adaptations over time. By using an integrative multidisciplinary approach, immunomics offers enormous potential to advance $21^{\text {st }}$ century antigen discovery and rational vaccine design against complex pathogens such as the Plasmodium parasite.

Key words: Immunomics, immunome, antigen discovery, rational vaccine design, vaccine development, parasites, malaria, complex pathogens.

\section{INTRODUCTION}

The shift from an empirical to a rational method for vaccine development associated with an enhanced understanding of host-pathogen interactions is synergistic with advances in genomics and bioinformatics. Since the first complete sequencing of a DNA genome over 20 years ago, that of the phage $\Phi-\mathrm{X} 174$, with only 5368 base pairs (Sanger et al. 1977), the number of sequenced genomes, proteomes and transcriptomes of different pathogens has risen exponentially. There are now over 6500 complete genomes published from over 59000 organisms, representing bacteria, viruses, parasites and eukaryotes, as available on the GOLD database (https://gold.jgi-psf.org/index). This includes the genomes, proteomes and/or transcriptomes of human, nonhuman primate, and rodent species of Plasmodium. This wealth of information is a direct consequence of technological advances focused at the molecular level and allows for multidisciplinary approaches to identify target antigens for the development of vaccines, drugs and diagnostic tests, and for the development and application of methods to identify immune correlates of protection (Doolan et al. 2014). Complementary methods, such as next-generation sequencing of lymphocyte epertoires (Mehr, 2014), complete T- and B-cell phenotype analysis (Zarnitsyna et al. 2013), highly-

* Corresponding author. QIMR Berghofer Medical Research Institute, Locked Bag 2000 Royal Brisbane Hospital, QLD 4029, Australia. E-mail: Denise. Doolan@qimrberghofer.edu.au sensitive gene expression measurement using Fluidigm (Spurgeon et al. 2008) or Nanostring (Geiss et al. 2008), high-throughput profiling technologies using CyTOF mass cytometer (Kidd et al. 2014; Hansmann et al. 2015), and biophotonic imaging for visualizing the infectious disease process (Andreu et al. 2011), among other advances, have the potential to enhance understanding of the interaction between host and pathogens at the molecular level. The availability and refinement of large-scale bioinformatic databases containing information on both host and pathogen can further advance the acquisition, analysis and application of research data to yield more clinically relevant outcomes, ideally leading to the development of vaccines that provide sterile life-long protective immunity without the need for boosting, or sensitive and specific biomarkers of pathogens exposure or protective immunity. Such applications are highly desirable in the malaria community.

The term 'immunomics' was coined in 2001 by Klysik (Klysik, 2001), who suggested that ongoing advances in technology should serve to address the correlations between genes and the functional properties of their protein products. Today, the term immunomics refers to an integration of molecular immunology, genomics, proteomics, transcriptomics and bioinformatics, effectively providing a much-needed link between these fields (Doolan, 2011) and enabling an effective correlation between immunology research and clinical application. Immunomics is the study of the immunome, which can be defined as the set of antigens or 
epitopes that interface with the host immune system (Sette et al. 2005). However, it is necessary to consider that the definition of immunomics might be subject to different interpretations, as usually happens with the fast-changing fields of study like the ones comprised in the general domain of omics, and is likely to expand and embrace concepts that might still be under investigation. Thus, the evolution of methods and techniques in the fields that are currently under the concept of immunomics will likely influence the definition and context of this term. In this paper, we are using the Sette et al. definition for immunomics.

Immunomics is distinct from reverse vaccinology, systems immunology and vaccinomics. Reverse vaccinology aims to identify the complete repertoire of antigens that an organism is capable of secreting or expressing on its surface (Rinaudo et al. 2009). Systems immunology is a sub-discipline of systems biology (Schubert, 2011), and deals with molecular mechanisms of how the components of the immune system work together as a whole (Narang et al. 2012). Vaccinomics, on the other hand, integrates immunogenetics and immunogenomics with systems biology and immune responses (Poland et al. 2011), Aimed at creating vaccines that are personalized/individualized.

Immunomics is as dependent on the host as it is on the pathogen, since the immune system and the infecting pathogens have been co-evolving for thousands of years. Furthermore individual characteristics of the parasite (e.g. species, strain, virulence, etc) interact with individual characteristics of the host immune system (e.g. age, immune status, epigenetic traits) (Tournier and Quesnel-Hellmann, 2006; Stilling et al. 2014). Each of the components of the immune response is extremely complex on its own, and the interactions between them create an even more complex network of reactions. This complexity creates a challenge for in-depth, comprehensive analyses and increases the cost of experimental verification. An immunomics-based approach offers a solution to this challenge since highthroughput screening is performed (at least in part) in silico, prior to in vitro and in vivo experimental verification. High-throughput screening is used intensely (and successfully) for lead and drug discovery (Balls et al. 2012; Annang et al. 2015). Translation of this technique to immunomics in order to address the challenge created by the complexity of the immune response to a pathogen includes the use of powerful computational analysis of next-generation, high-density peptide microarrays for rapid discovery and mapping of antigenic determinants (Hecker et al. 2012; Carmona et al. 2015). This allows for a more thorough, unbiased and rational approach. In this fashion, data-driven studies of the immunome facilitate identification and characterization of key antigens and epitopes.

\section{TRADITIONAL VACCINOLOGY}

Pathogens with complex life cycles, complex genomes, proteomes and transcriptomes, and correspondingly complex immunomes, represent a great challenge for the development of effective vaccines. The Plasmodium parasite which causes malaria exemplifies this challenge. Such pathogens express a broad repertoire of antigens and epitopes that could be available for recognition by the host immune system. In some cases, effective immune responses directed against only a subset of these antigens and epitopes are sufficient for competent protection. This is demonstrated by the effectiveness of subunit vaccines such as the recombinant $\mathrm{HBsAg}$ antigen based vaccine for hepatitis B (Arnon, 2011; Schetgen, 2014). However, for many pathogens, subunit vaccines based on only one or a few antigens have proved poorly effective (Meeusen et al. 2007; Foged et al. 2014). For many vaccines that are currently in use worldwide, for example the highly effective whole-organism based Bacillus Calmette-Guérin (BCG) vaccine, the mechanism of protective immunity remains unknown (Kaufmann et al. 2014). This gap in knowledge highlights the difficulties in assessing clear interactions between host and pathogens. Due to the poor efficacy of most subunit vaccines, until very recently almost all licensed vaccines were based on the whole organism, typically either live-attenuated pathogens or inactivated/killed pathogens (Grimm and Ackerman, 2013). In the case of malaria where extensive efforts directed towards subunit vaccines have thus far failed (Schwartz et al. 2012), a resurgence of effort towards development of a whole-organism vaccine has occurred (Hoffman et al. 2010; McCarthy and Good, 2010; Good, 2011; Mikolajczak et al. 2014). A genetically attenuated Trypanosoma cruzi parasite is also being considered for vaccination against Chagas disease (SanchezValdez et al. 2015). However, these whole-organism vaccines are associated with number of problems. In some cases, they have been associated with reversion to virulence, causing a proportion of vacinees to develop some form of the disease they had been vaccinated against (Bonanni and Santos, 2011). Furthermore, whole-organism vaccines are almost exclusively restricted to pathogens that can be cultured in vitro and have relatively low antigen variability, which can be difficult for pathogens like Plasmodium (malaria), Mycobacterium tuberculosis (tuberculosis) or Schistosoma (schistosomiasis) (Doolan et al. 2014).

\section{Reverse vaccinology for rational vaccine design}

An alternative strategy to culture-based in vitro approaches for the development of an effective vaccine against complex pathogens is that of reverse vaccinology, pioneered by Rappuoli in 2001 (Rappuoli, 2001). This approach analyses the entire 
genome of a pathogen to rapidly identify putative protective antigens and predict potential vaccine candidates (Rinaudo et al. 2009; Heinson et al. 2015). Reverse vaccinology studies were among the first to harness the wealth of information generated by genome sequencing for vaccine development. The proof of concept for this approach was established by the screening of surface-exposed proteins in the Neisseria meningitides genome, a causative agent of meningococcal disease, and resulted in a phase III vaccine trial after 40 years of setbacks using conventional methods (Pizza et al. 2000; Serruto et al. 2012). Briefly, the sequence of the $N$. meningitides virulent strain MC58 was analysed using bioinformatics algorithms for surface-exposed proteins, which were then recombinantly expressed in $E$. coli, purified and tested in mice for their potential to induce bactericidal antibodies. Humoral response was analysed by Western blot and surface localization of the target protein was confirmed by enzyme-linked immunosorbent assay (ELISA) and flow cytometry. Of the 91 proteins found to be positive in the bioinformatic screening, 28 were able to induce antibodies with bactericidal activity and were prioritized based on their ability to induce broad protection. Ultimately five proteins were combined in a multicomponent vaccine named 4CMenB (Pizza et al. 2000; Serruto et al. 2012). Very recently, two multicomponent vaccine formulations based on sequencing of the whole meningococcal genome to identify surface antigens of the meningococcal strains, called Bexsero $^{\circledR}$ (Novartis) and Trumemba ${ }^{\mathrm{TM}}$ (Pfizer), were made available for clinical immunization against invasive group B meningococcal disease, although still subject to additional monitoring; however, unfavourable cost-effectiveness ratios for application of these vaccines have been reported (Christensen et al. 2013; Pouwels et al. 2013; Leca et al. 2015; Tirani et al. 2015).

Reverse vaccinology has limitations; in particular, it cannot predict polysaccharides, lipids or glycolipids which may be active compounds for a vaccine (Kanampalliwar et al. 2013; Bertholet et al. 2014). It is also unlikely that a reverse vaccinology approach by itself to be able to predict a good correlate of protective immunity (Bertholet et al. 2014).

\section{IMMUNOMICS-BASED VACCINOLOGY}

In a field of study as complex as vaccinology, where the intricacy of the human immune system is evident from the cohorts of non-homogenous groups where disease phenotype and molecular profile (among other elements) have immense variation (Falus, 2008), elegant approaches are required to decipher the host immune response to pathogens. Immunomics facilitates such a rational, systematic and comprehensive approach to antigen selection and prioritization for vaccine development. The wealth of information that immunomics draws on, namely large-scale genomic, proteomic and transcriptomic datasets, can be accessed via large public-access databanks such as GenBank (http:// www.ncbi.nlm.nih.gov/genbank) or UniProt (http://www.uniprot.org/); pathogen-specific databases such as PlasmoDB (www.PlasmoDB.org) or TriTrypDB (http://tritrypdb.org/tritrypdb); or immunology based databases such as the Immune Epitope Database (http://www.iedb.org/) or the Innate Immune Database (http://www.innatedb. com). The available information in these databases is used as primary input for epitope prediction and therefore it is crucial that sequences are verified, annotated and curated. Moreover, immunomics also faces the challenges associated with computational predictive algorithms, which are subject to a series of conditional instructions for weightings and outputs dependent on 'learned' behaviour or characteristics. If those conditional instructions are flawed, the error is magnified exponentially, resulting in inaccurate data.

However, the multifactorial nature of protective immunity (Pulendran and Ahmed, 2006), the large number of antigenic determinants or epitopes that can be recognized by the cells of the immune system as immunodominant or subdominant epitopes (Sette and Sundaram, 2006), the broad range of putative epitopes restricted by multiple human leukocyte antigen (HLA) alleles prevalent in the human population (del Guercio et al. 1995) combined with the high variation in the frequencies of different major histocompatibility complexes (MHC) alleles in different ethnicities (Sidney et al. 2010), precludes analysing such large and complex datasets without the use of computation. The need for accurate prediction of biologically relevant epitopes for rational vaccine design is therefore crucial.

\section{Computational predictive methods}

The prediction of peptide epitopes from primary protein sequences is not a modern achievement. In fact, reports from as early as 1987 show that peptide epitopes could be predicted from protein sequence by consideration of hydrophobicity and amphipathic helices, and these sequences could be synthesized to enable further study (Cease et al. 1987; Gotch et al. 1987; Margalit et al. 1987). Subsequently, it was established that $\mathrm{T}$ cell epitopes are generally linear and continuous. MHC class I molecules typically bind peptides that are 8-15 amino acids long (Rammensee et al. 1999), while MHC class II molecules usually bind longer peptides of 12-25 amino acids in length (Jardetzky et al. 1996). These features provided the foundation for improved prediction algorithms that considered the affinity of binding of a specific peptide sequence to a given MHC molecule. Using experimental 
affinity data deposited in public databases as training data, researchers developed statistical methods to take the early prediction algorithms to a new level. Widely used algorithms include those in the Immune Epitope DataBase (Kim et al. 2012; Vita et al. 2015) such as average relative binding (ARB) (Bui et al. 2005), or alternate algorithms such as support vector machine for prediction of $\mathrm{MHC}$ binding peptides (SVMHC) (Donnes and Kohlbacher, 2006) or NetMHCII-2·2 (Nielsen and Lund, 2009). More recent prediction algorithms consider additional features, such as proteosomal cleavage sites and transporter associated with antigen processing (TAP)-binding patterns, further enhancing accuracy (Tenzer et al. 2005; Antonets and Bazhan, 2013). Current computational models consider quantitative matrices, artificial neural networks, hidden Markov models, support vector machines (SVMs), quantitative structure activity relationship and molecular docking simulations (Brusic et al. 2004; Desai and Kulkarni-Kale, 2014). These improvements are crucial given the high degree of MHC polymorphism and complexity of generation and presentation of $\mathrm{T}$ cell epitopes (Desai and Kulkarni-Kale, 2014). Overall, in silico epitope predictions represent a more targeted, costand time-effective strategy as compared with more traditional approaches such as screening pools of overlapping peptide pools by enzyme-linked immunospot (ELIspot) or intracellular cytokine staining (ICS), or other epitope identification methods like $\mathrm{X}$-ray crystallography and nuclear magnetic ressonance (NMR) techniques (Sun et al. 2013).

Physicochemical properties associated with the $\mathrm{T}$ cell receptor also critically influence effective cell mediated immune responses (Osuna et al. 2014, Madura et al. 2015). Very recent studies have shown that the $\mathrm{T}$ cell receptor (TCR) undergoes conformational changes upon engagement with a peptide, allowing for discrimination between peptides. This conformational change causes the peptide to diverge its amino terminus partly away from the MHC peptide binding groove, forming a higher affinity interface with the TCR than is formed with the MHC groove (Dyson, 2015; Madura et al. 2015). This discovery suggests that future epitope predictions strategies that consider details such as antigen TCR-MHC affinity may result in improved accuracy of epitope predictions and consequently reduced time for validation studies.

With regard to B-cell epitope predictions, most of the computational methods and databases currently available focus on continuous or linear B cell epitopes (Ansari and Raghava, 2010). However, many B-cell epitopes are conformational and discontinuous (Braga-Neto and Marques, 2006; Ansari and Raghava, 2010), corresponding to the tridimensional features on the surface of the antigen where recognition by the immune system occurs (BragaNeto and Marques, 2006). This creates difficulties for the bioinformatic prediction of B-cell epitopes. Ideally, predictive algorithms would use tridimensional surface models of the protein antigens and measure surface energy interactions of variable regions of the immunoglobulins that correlate with B-cell activation. However, due to the computational complexity associated with analysing tridimensional interactions and the limited number of known antibody-antigen complex structures, only a limited number of prediction methods exist for discontinuous epitopes [reviewed in (Yao et al. 2013)]; most are considered to perform poorly (Sun et al. 2013). It has been suggested that combining multiple classifiers for B-cell epitope definition, as an ensemble, could improve the performance of computational B-cell epitope prediction tools (E1Manzalawy and Honavar 2014), but further multidisciplinary understanding of conformational epitopes may also contribute to the improving performance of these predictive algorithms.

\section{Enabling technologies}

Immunomics-based approaches can enhance our understanding of key features of the immune system in health and disease. Classical methods of assessing immune responses have typically focused only on the frequency and magnitude of a single immune parameter, e.g. antibody titre. Immunomics, on the other hand, allows for a multifactorial view of the response and considers the relevant biological outcome, e.g. protective immunity, by effectively taking into account pertinent and appropriate elements, such as particular epitope combinations, the cytokine response measured, and the $\mathrm{T}$ cell population used as the target. Multiple experimental methods, with different strengths, must also be used, and consequent results integrated. For example, using an immunomics approach to a disease model, Quintana and his team have shown that immunomics can be used to predict future disease (Quintana et al. 2004). Moreover, it has been computationally shown how the immune response to an epitope modulates the behaviour of an immune network, providing evidence for immunomic regulatory networks from immunomic microarray data (Braga-Neto and Marques, 2006).

The quality of the immune response is at least as important as the quantity, as quality can be a key determinant of protection (Zepp, 2010; Doolan et al. 2014). Some of the more conventional methods for assessment of immune responses as a measure of Tor B-cell reactivity include immunoassays such as the Jerne plaque assay (Jerne and Nordin 1963), the splenic focus assay (Klinman and Aschinazi 1971), ELISA (Engvall and Perlmann 1971), interferon- $\gamma$ (IFN- $\gamma$ ) ELIspot (Czerkinsky et al. 1983), 
fluorescence-activated cell sorting (Hayakawa et al. 1987; McHeyzer-Williams et al. 1993), peptideinduced ICS (Ozen et al. 1998), and tetramer staining (Altman et al. 1996; Skinner et al. 2000). More recently, technological and conceptual advances have resulted in the development and application of novel methods to comprehensively assess immune responses, including approaches to simultaneously examine a large number of cell functions and phenotypic markers, including at the single cell level. Such methods include, but are not limited to, the analysis of gene expression using Nanostring (Geiss et al. 2008) or Fluidigm (Spurgeon et al. 2008), as well as mass spectrometry based methods such as CyTOF technologies (Cheung and Utz, 2011). The quality of those responses can be assessed via phenotypic markers, differentiation state, profile of secreted cytokines, avidity, affinity and repertoire diversity (Siegrist, 2013). If used singularly, none of the methods cited above can describe the complete set of characteristics that define an antigen-specific response. Immunomics can address such problems by integrating different fields of study, thus providing a platform for combining the strengths and compensating the flaws of different methods and approaches to vaccine development.

Particularly transformative are multidisciplinary computational and mathematical methods developed to cope with multiplex data, including host-pathogen interactions (Raman et al. 2010), for which experimental analysis may be costly and laborious if the pathogen is complex. These mathematical and bioinformatic platforms may also support other aspects of vaccinology such as adjuvant discovery (Schellhammer and Rarey, 2004; Sollner et al. 2010).

\section{Immunomics for rational vaccine design}

The use of an immunomics based approach to vaccinology is a promising alternative for efficacious vaccine design. Unlike reverse vaccinology, immunomics also considers the immune system. It provides a means to systematically identify the antigens and epitopes that interact with the host immune system (Sette et al. 2005; De Groot, 2006). The premise of an immunomics-based, rational vaccine design is a consistent induction of the desired immune response against the key pathogen antigen(s) or epitopes which are targeted by protective immune responses (Barbosa and BarralNetto 2013; Doolan et al. 2014; Slifka and Amanna, 2014).

Immunomics of viruses. A number of immunomics approaches have been applied to viruses. In the case of HIV, an effective vaccine might require the design or discovery of immunogens which elicit good neutralizing antibodies against circulating strains of the virus (Kwong et al. 2011). For many years, only a few neutralizing monoclonal antibodies against the virus were known; the most intensely studied have been the antibodies directed against the glycoproteins gp120 and gp41 (Burton et al. 2012). With the advent of immunomics, more neutralizing monoclonal antibodies, some with unexpected epitopes, have been identified (see Burton et al. 2012). Most recently, Gallerano et al. (2015) have analysed polyclonal antibody responses of HIV-infected persons to overlapping peptides covering the complete amino acid sequences of the gp120 and gp41 proteins, and identified major epitopes that can be recognized by antibodies.

Another immunomics study has prospected the influenza A (H1N1) virus immunome and transcriptome to show a complex host response pathway to the virus, and unravelled interactions between virus and host (Dimitrakopoulou et al. 2014). A different study has used immunomics to better understand the repertoire of $\mathrm{T}$ cell specificities for H1N1, with the objective of developing an universal vaccine for influenza virus to combat the continuous antigenic drift of the virus (Assarsson et al. 2008). This study used over 4000 peptides from a panel of 23 influenza A virus strains based on predicted high-affinity binding to HLA class I or class II and high conservancy levels. Peripheral blood mononuclear cells (PBMCs) from healthy human donors were tested for reactivity against HLA-matched peptides by using IFN- $\gamma$ ELIspot. One epitope, called PB1, was found to be the major target for both $\mathrm{CD}^{+}$and $\mathrm{CD}^{+}{ }^{+} \mathrm{T}$ cell responses; 54 other non-redundant epitopes (38 class I and 16 class II) were also identified, and provide a potential base for the development of a universal influenza vaccine (Assarsson et al. 2008).

Immunomics of bacteria. Immunomics has been also successfully applied to bacteria. In one example, a Francisella tularensis protein microarray was generated and probed with serum from experimentally immunized mice to identify 11 of the 12 antigens previously discovered using traditional methods plus an additional 31 new antigens (Eyles et al. 2007); this study further demonstrated an $\operatorname{IgG}$ subclass bias towards $\operatorname{IgG} 2 \mathrm{a}$ in protected animals.

T cell based approaches have also been pursued to advance bacterial vaccine development. Moise $e t$ al. (Moise et al. 2012) evaluated the Helicobacter pylori genome for $\mathrm{CD}^{+} \mathrm{T}$ cell epitopes by using the predictor algorithm EpiMatrix; resulting epitopes were experimentally validated for MHC binding and $\mathrm{T}$ cell reactivity in p27 knockout mice infected with the mouse-adapted $H$.pylori strain. The immunoreactive epitopes were assembled into a multiepitope vaccine that induced a broad immune response as determined by IFN- $\gamma$ production in ELIspot assays (Moise et al. 2012). 
For Mycobacterium tuberculosis, $\mathrm{CD}^{+} \mathrm{T}$ cells are crucial for controlling the infection (Woodworth et al. 2014). Recently, a genome-wide screening for $\mathrm{CD}^{+} \mathrm{T}$ cell reactivity against $M$. tuberculosis (Arlehamn et al. 2013) identified a number of novel epitopes and antigens that may represent a potential vaccine candidates. This immunomics-based study screened the sequences from five complete $M$. tuberculosis genomes available from the National Center for Biotechnology Information (NCBI) database and protein sequences were parsed into 15-mer peptides, which were then ranked by consensus percentile of HLA binding for the 22 alleles most commonly present in the general population. For each protein, no less than two of the best-predicted binders were selected for synthesis, creating a synthetic library of over twenty thousand peptides that were tested by IFN- $\gamma$ ELIspot against $\mathrm{T}$ cells from latent TBinfected donors. The reactive epitopes were ranked on the basis of magnitude of response and mapped to individual bacterial antigens using a reference genome. The results revealed a very heterogeneous response to infection: 82 antigens were recognized by more than $10 \%$ of donors. Hundreds of novel epitopes recognized by the human immune system have been identified, attesting to the potential of genomewide screening strategy (Arlehamn et al. 2013; Arlehamn and Sette, 2014).

Immunomics of parasites. We and others have had a particular interest in the immunomics of Plasmodium spp. (Doolan, 2011). In early studies which served as proof of concept for the protein microarray platform, 250 genes representing putative proteins from $P$. falciparum were selected from a genomic sequence database according to the pattern of stage-specific gene or protein expression, subcellular localization, secondary structure, and known immunogenicity or antigenicity in human and animal models (Doolan et al. 2008). Each of these 250 sequences was then printed onto a protein microarray which was probed with human sera from individuals differing in immune status. This study showed that the protein microarray platform could be successfully applied to identify antigens recognised as serodominant by individuals naturally or experimentally exposed to malaria. A more comprehensive array of 2320 protein fragments representing $23 \%$ of the $P$. falciparum proteome was then fabricated; the protein selection considered stage-specific transcription or protein expression, subcellular localization, secondary protein structure, and documented immunogenicity in humans or animal models at the time of antigen selection, as indicated by multidimensional protein identification technology. Subsequent studies with this array identified a signature of 16 proteins that were associated with the sterile immunity induced by experimental immunization with radiation attenuated sporozoites (Trieu et al. 2011), and a signature of 49 antigens associated with the anti-disease immunity induced by natural exposure to malaria (Crompton et al. 2010). Those data provide experimental support for a multivalent vaccine. These and other studies identified a number of antigens that had not been previously described as immunologically reactive (reviewed in (Davies et al. 2015)). Our group has also shown that immunomics-based approaches can be applied to the study of cellular responses against Plasmodium. In the proof of concept demonstration of an epitope-based $\mathrm{T}$ cell screening approach in the $P$. falciparum model, the parasite genomic sequence was scanned to identify and prioritize a set of genes representing antigens potentially expressed in the sporozoite and intrahepatic stage of the parasite life cycle. A total of 27 proteins putatively expressed in the sporozoite proteome were selected according to their level of expression in the sporozoite proteome as determined by MudPIT, as well as stage specificity. This panel included 10 antigens expressed only in sporozoites, and 17 antigens common to other stages of the parasite life cycle. Evaluation of these proteins has shown that 16 of them were reproducibly recognised by peripheral blood mononuclear cell (PBMC)s from irradiated sporozoite immunized volunteers but not by naive controls, and nine of these antigens were more antigenic than other well-characterized antigens considered as leading vaccine candidates (Doolan et al. 2003). Subsequently, we have applied this strategy to the complete pre-erythrocytic stage proteome and shown that only approximately $30 \%$ of the proteome is recognized, and identified the set of antigens that are highly reactive for $\mathrm{T}$ cell responses (Proietti \& Doolan, in preparation).

With regard to immunomics for other parasites, Schistosoma proteome and transcriptome have been mined to identify surface-derived proteins, a subset of which were then expressed and printed on a protein microarray (Driguez et al. 2010; Loukas et al. 2011). These arrays have been probed with specimens from humans representing distinct clinical categories as well as experimentally immunized rodents. Several proteins which are predicted to be good potential vaccine targets have been identified (Loukas et al. 2011; McWilliam et al. 2012). These include the tetraspanin SmTSP-2, the tegumental antigen Sm29, and the very low-density lipoprotein-binding protein SjSVLBP as well as other novel proteins (reviewed in (McWilliam et al. 2012). More recently, these protein microarrays have been screened for IgE and multiple IgG subclasses responses using sera from resistant or susceptible individuals (Gaze et al. 2014). The resultant antibody profiles could distinguish between protected $v s$ non-protected cohorts and allowed identification of antigens that might represent excellent vaccine candidates (Gaze et al. 2014). The same 
study also identified protein associated with potentially deleterious hypersensitivity responses if used as subunit vaccines in endemic populations.

\section{CONCLUSION}

Infectious diseases continue to pose a major threat to public health worldwide and the need for prophylactic or therapeutic vaccines is urgent. Many of the diseases with high indexes of mortality or morbidity are caused by pathogens with large complex genomes and multistage life cycles, which present substantial challenges for the development of an effective vaccine. Immunomics, by focusing on the key components of host-pathogen interactions, provides a sound foundation to systematically search for critical determinants of immunity, namely key target antigens and epitopes, which could form the base of rationally designed new generation vaccines. Provided that immunomics continues to exploit state-of-theart techniques and technologies and is able to respond to the inherent challenges associated with large datasets, this approach offers, in our opinion, an enormous potential as a 21 st century solution to the challenge of rationally designing vaccines which are highly effective against complex pathogens such as the causative agent of malaria.

\section{ACKNOWLEDGEMENTS}

KdS is supported by the International Postgraduate Research Scholarship from the University of Queensland. DLD is supported by a National Health and Medical Research Council (NHMRC) Principal Research Fellowship; support by a Pfizer Australia Senior Research Fellowship is also gratefully acknowledged.

\section{F INANCIAL SUPPORT}

This research received no specific grant from any funding agency, commercial or not-for-profit sectors.

\section{REFERENCES}

Altman, J. D., Moss, P. A., Goulder, P. J., Barouch, D. H., McHeyzerWilliams, M. G., Bell, J. I., McMichael, A. J. and Davis, M. M. (1996) Phenotypic analysis of antigen-specific T lymphocytes. Science 274, 94-96. Andreu, N., Zelmer, A. and Wiles, S. (2011). Noninvasive biophotonic imaging for studies of infectious disease. FEMS Microbiology Review 35, 360-394.

Annang, F., Perez-Moreno, G., Garcia-Hernandez, R., CordonObras, C., Martin, J., Tormo, J. R., Rodriguez, L., de Pedro, N., Gomez-Perez, V., Valente, M., Reyes, F., Genilloud, O., Vicente, F., Castanys, S., Ruiz-Perez, L. M., Navarro, M., Gamarro, F. and Gonzalez-Pacanowska, D. (2015). High-throughput screening platform for natural product-based drug discovery against 3 neglected tropical diseases: human African trypanosomiasis, leishmaniasis, and Chagas disease. Fournal of Biomolecular Screening 20, 82-91.

Ansari, H. R. and Raghava, G. P.S. (2010). Identification of conformational B-cell epitopes in an antigen from its primary sequence. Immunome Research 6, 6-6.

Antonets, D. V. and Bazhan, S. I. (2013). PolyCTLDesigner: a computational tool for constructing polyepitope T-cell antigens. BMC Research Notes 6, 1756-0500.
Arlehamn, C. S. L. and Sette, A. (2014). Definition of CD4 immunosignatures associated with MTB. Frontiers in Immunology 5, 124.

Arlehamn, C.S.L., Gerasimova, A., Mele, F., Henderson, R., Swann, J., Greenbaum, J.A., Kim, Y., Sidney, J., James, E. A., Taplitz, R., McKinney, D. M., Kwok, W. W., Grey, H., Sallusto, F., Peters, B. and Sette, A. (2013). Memory T cells in latent Mycobacterium tuberculosis infection are directed against three antigenic islands and largely contained in a CXCR3 + CCR6 + Th1 subset. PLoS Pathogens 9, 24.

Arnon, R. (2011). Overview of vaccine strategies. In Vaccine Design: Innovative Approaches and Novel Strategies (ed. Rappuoli, R. and Bagnoli, F.), pp. 1-20. Caister Academic Press, Norfolk, UK.

Assarsson, E., Bui, H. H., Sidney, J., Zhang, Q., Glenn, J., Oseroff, C., Mbawuike, I. N., Alexander, J., Newman, M. J., Grey, H. and Sette, A. (2008). Immunomic analysis of the repertoire of T-cell specificities for influenza A virus in humans. Fournal of Virology 82, 12241-12251. Balls, M., Bennet, A. and Kendall, D. (2012). Translation of new technologies in biomedicines: shaping the road from basic research to drug development and clinical application - and back again. In Pharmaceutical Biotechnology: Drug Discovery and Clinical Applications (ed. Kayser, O. and Warzecha, H.), pp. 113-152. Wiley-Blackwell, Weinheim, Germany. Barbosa, T. and Barral-Netto, M. (2013). Challenges in the research and development of new human vaccines. Brazilian Fournal of Medical and Biological Research 46, 103-108.

Bertholet, S., Reed, S. G. and Rappuoli, R. (2014). Reverse vaccinology. In The Art $\mathcal{O}^{\circ}$ Science of Tuberculosis Vaccine Development (ed. Nor, N., Acosta, A. and Sarmiento, M. E.), Chapter 3.1. Oxford University Press, Selangor Darul Ehsan, Malaysia. http://tbvaccines.usm.my/finlay/? $\mathrm{q}=$ content/chapter-31-reverse-vaccinology.

Bonanni, P. and Santos, J. I. (2011). Vaccine evolution. Perspectives in Vaccinology 1, 1-24.

Braga-Neto, U. and Marques, E. (2006). From functional genomics to functional immunomics: new challenges, old problems, big rewards. PLoS Computational Biology 2, e81.

Brusic, V., Bajic, V.B. and Petrovsky, N. (2004). Computational methods for prediction of T-cell epitopes - a framework for modelling, testing, and applications. Methods 34, 436-443.

Bui, H. H., Sidney, J., Peters, B., Sathiamurthy, M., Sinichi, A., Purton, K. A., Mothe, B. R., Chisari, F.V., Watkins, D. I. and Sette, A. (2005). Automated generation and evaluation of specific MHC binding predictive tools: ARB matrix applications. Immunogenetics 57, 304-314.

Burton, D. R., Poignard, P., Stanfield, R. L. and Wilson, I. A. (2012). Broadly neutralizing antibodies present new prospects to counter highly antigenically diverse viruses. Science 337, 183-186.

Carmona, S. J., Nielsen, M., Schafer-Nielsen, C., Mucci, J., Altcheh, J., Balouz, V., Tekiel, V., Frasch, A. C., Campetella, O., Buscaglia, C. A. and Aguero, F. (2015). Towards high-throughput immunomics for infectious diseases: use of next-generation peptide microarrays for rapid discovery and mapping of antigenic determinants. Molecular \& Cellular Proteomics 28, 045906.

Cease, K. B., Margalit, H., Cornette, J. L., Putney, S. D., Robey, W. G., Ouyang, C., Streicher, H. Z., Fischinger, P. J., Gallo, R. C. and DeLisi, C. (1987). Helper T-cell antigenic site identification in the acquired immunodeficiency syndrome virus gp120 envelope protein and induction of immunity in mice to the native protein using a 16-residue synthetic peptide. Proceedings of the National Academy of Sciences of the United States of America 84, 4249-4253.

Cheung, R. K. and Utz, P. J. (2011). Screening: CyTOF-the next generation of cell detection. Nat Rev Rheum 7, 502-503.

Christensen, H., Hickman, M., Edmunds, W. J. and Trotter, C. L. (2013). Introducing vaccination against serogroup B meningococcal disease: an economic and mathematical modelling study of potential impact. Vaccine 31, 2638-2646.

Crompton, P.D., Kayala, M.A., Traore, B., Kayentao, K., Ongoiba, A., Weiss, G. E., Molina, D. M., Burk, C. R., Waisberg, M., Jasinskas, A., Tan, X., Doumbo, S., Doumtabe, D., Kone, Y., Narum, D. L., Liang, X., Doumbo, O. K., Miller, L. H., Doolan, D. L., Baldi, P., Felgner, P. L. and Pierce, S. K. (2010). A prospective analysis of the Ab response to Plasmodium falciparum before and after a malaria season by protein microarray. Proceedings of the National Academy of Sciences of the United States of America 107, 6958-6963.

Czerkinsky, C., Nilsson, L.-Å., Nygren, H., Ouchterlony, Ö. and Tarkowski, A. (1983). A solid-phase enzyme-linked immunospot (ELISPOT) assay for enumeration of specific antibody-secreting cells. fournal of Immunological Methods 65, 109-121.

Davies, D. H., Duffy, P., Bodmer, J.-L., Felgner, P. L. and Doolan, D. L. (2015). Large screen approaches to identify novel malaria vaccine candidates. Vaccine. 
De Groot, A. S. (2006). Immunomics: discovering new targets for vaccines and therapeutics. Drug Discovery Today 11, 203-209.

del Guercio, M. F., Sidney, J., Hermanson, G., Perez, C., Grey, H. M., Kubo, R. T. and Sette, A. (1995). Binding of a peptide antigen to multiple HLA alleles allows definition of an A2-like supertype. Fournal of Immunology 154, 685-693.

Desai, D. V. and Kulkarni-Kale, U. (2014). T-cell epitope prediction methods: an overview. Methods Mol Biol. 1184, 333-364.

Dimitrakopoulou, K., Dimitrakopoulos, G. N., Wilk, E., Tsimpouris, C., Sgarbas, K. N., Schughart, K. and Bezerianos, A. (2014). Influenza A immunomics and public health omics: the dynamic pathway interplay in host response to H1N1 infection. Omics 18, 167-183. Donnes, P. and Kohlbacher, O. (2006). SVMHC: a server for prediction of MHC-binding peptides. Nucleic Acids Research 34, W194-W197.

Doolan, D. L. (2011). Plasmodium immunomics. International fournal of Parasitology 41, 3-20.

Doolan, D. L., Southwood, S., Freilich, D. A., Sidney, J., Graber, N. L., Shatney, L., Bebris, L., Florens, L., Dobano, C., Witney, A. A., Appella, E., Hoffman, S. L., Yates, J. R. 3rd, Carucci, D. J. and Sette, A. (2003). Identification of Plasmodium falciparum antigens by antigenic analysis of genomic and proteomic data. Proceedings of the National Academy of Sciences of the United States of America 100, 99529957.

Doolan, D. L., Mu, Y., Unal, B., Sundaresh, S., Hirst, S., Valdez, C., Randall, A., Molina, D., Liang, X., Freilich, D. A., Oloo, J. A., Blair, P. L., Aguiar, J. C., Baldi, P., Davies, D. H. and Felgner, P. L. (2008). Profiling humoral immune responses to $P$. falciparum infection with protein microarrays. PROTEOMICS 8, 4680-4694.

Doolan, D. L., Apte, S. H. and Proietti, C. (2014). Genome-based vaccine design: the promise for malaria and other infectious diseases. International fournal for Parasitology 44, 901-913.

Driguez, P., Doolan, D. L., Loukas, A., Felgner, P. L. and

McManus, D. P. (2010). Schistosomiasis vaccine discovery using immunomics. Parasites \& Vectors 3, 1756-3305.

Dyson, J. (2015). T-cell receptors: tugging on the anchor for a tighter hold on the tumor-associated peptide. European Fournal of Immunology 45, 380-382. El-Manzalawy, Y. and Honavar, V. (2014). Building classifier ensembles for B-Cell epitope prediction. Methods in Molecular Biology (Clifton, N.F.) 1184, 285-294

Engvall, E. and Perlmann, P. (1971). Enzyme-linked immunosorbent assay (ELISA). Quantitative assay of immunoglobulin G. Immunochemistry 8, 871-874.

Eyles, J. E., Unal, B., Hartley, M. G., Newstead, S. L., FlickSmith, H., Prior, J. L., Oyston, P. C., Randall, A., Mu, Y., Hirst, S., Molina, D. M., Davies, D. H., Milne, T., Griffin, K. F., Baldi, P., Titball, R. W. and Felgner, P. L. (2007). Immunodominant Francisella tularensis antigens identified using proteome microarray. PROTEOMICS 7, 2172-2183.

Falus, A. (2008). Clinical Applications of Immunomics, Springer Science \& Business Media, New York

Foged, C., Rades, T., Perrie, Y. and Hook, S. (2014). Subunit Vaccine Delivery, Springer, New York.

Gallerano, D., Ndlovu, P., Makupe, I., Focke-Tejk1, M., Fauland, K., Wollmann, E., Puchhammer-Stöck1, E., Keller, W., Sibanda, E. and Valenta, R. (2015). Comparison of the specificities of IgG, IgG-subclass, IgA and IgM reactivities in African and European HIV-infected individuals with an HIV-1 clade C proteome-based array. PLoS One 10, e0117204. doi: 10.1371/journal.pone.0117204

Gaze, S., Driguez, P., Pearson, M.S., Mendes, T., Doolan, D. L., Trieu, A., McManus, D. P., Gobert, G. N., Periago, M. V., Correa Oliveira, R., Cardoso, F.C., Oliveira, G., Nakajima, R., Jasinskas, A., Hung, C., Liang, L., Pablo, J., Bethony, J. M., Felgner, P. L. and Loukas, A. (2014). An immunomics approach to schistosome antigen discovery: antibody signatures of naturally resistant and chronically infected individuals from Endemic areas. PLoS Pathogens 10, e1004033.

Geiss, G. K., Bumgarner, R.E., Birditt, B., Dahl, T., Dowidar, N., Dunaway, D. L., Fell, H. P., Ferree, S., George, R. D., Grogan, T., James, J. J., Maysuria, M., Mitton, J. D., Oliveri, P., Osborn, J. L., Peng, T., Ratcliffe, A. L., Webster, P. J., Davidson, E. H., Hood, L. and Dimitrov, K. (2008). Direct multiplexed measurement of gene expression with color-coded probe pairs. Nature Biotechnology 26, 317-325. Good, M. F. (2011). A whole parasite vaccine to control the blood stages of Plasmodium: the case for lateral thinking. Trends in Parasitology 27, 335-340. Gotch, F., Rothbard, J., Howland, K., Townsend, A. and McMichael, A. (1987). Cytotoxic T lymphocytes recognize a fragment of influenza virus matrix protein in association with HLA-A2. Nature 326, 881-882.
Grimm, S. K. and Ackerman, M. E. (2013). Vaccine design: emerging concepts and renewed optimism. Current Opinion in Biotechnology 24, 1078-1088.

Hansmann, L., Blum, L., Hsin-Ju, C., Liedtke, M., Robinson, W. and Davis, M. M. (2015). Mass cytometry analysis shows that a novel memory phenotype B cell is expanded in multiple myeloma. Cancer Imm Res 3, 650660

Hayakawa, K., Ishii, R., Yamasaki, K., Kishimoto, T. and Hardy, R. (1987). Isolation of high-affinity memory B cells: Phycoerythrin as a probe for antigen-binding cells. Proceedings of the National Academy of Sciences of the United States of America 84, 1379-1383.

Hecker, M., Lorenz, P., Steinbeck, F., Hong, L., Riemekasten, G., Li, Y., Zettl, U. K. and Thiesen, H. J. (2012). Computational analysis of high-density peptide microarray data with application from systemic sclerosis to multiple sclerosis. Autoimmunity Reviews 11, 180-190.

Heinson, A. I., Woelk, C. H. and Newell, M. L. (2015). The promise of reverse vaccinology. International Health 7, 85-89.

Hoffman, S. L., Billingsley, P.F., James, E., Richman, A., Loyevsky, M., Li, T., Chakravarty, S., Gunasekera, A., Chattopadhyay, R., Li, M., Stafford, R., Ahumada, A., Epstein, J. E., Sedegah, M., Reyes, S., Richie, T. L., Lyke, K. E., Edelman, R., Laurens, M. B., Plowe, C. V. and Sim, B. K. (2010). Development of a metabolically active, non-replicating sporozoite vaccine to prevent Plasmodium falciparum malaria. Human Vaccines 6, 97-106.

Jardetzky, T. S., Brown, J. H., Gorga, J. C., Stern, L. J., Urban, R. G., Strominger, J. L. and Wiley, D. C. (1996). Crystallographic analysis of endogenous peptides associated with HLA-DR1 suggests a common, polyproline II-like conformation for bound peptides. Proceedings of the National Academy of Sciences of the United States of America 93, 734-738. Jerne, N. and Nordin, A. (1963). Plaque formation in agar by single antibody-producing cells. Science 140, 405.

Kanampalliwar, A., Rajkumar, S., Girdhar, A. and Archana, T. (2013). Reverse vaccinology: basics and applications. Fournal of Vaccines ङ Vaccination 4, 194-198

Kaufmann, S. H. E., Juliana McElrath, M., Lewis, D. J. M. and Del Giudice, G. (2014). Challenges and responses in human vaccine development. Current Opinion in Immunology 28, 18-26.

Kidd, B. A., Peters, L. A., Schadt, E. E. and Dudley, J. T. (2014). Unifying immunology with informatics and multiscale biology. Nature Immunology 15, 118-127.

Kim, Y., Ponomarenko, J., Zhu, Z., Tamang, D., Wang, P., Greenbaum, J., Lundegaard, C., Sette, A., Lund, O., Bourne, P. E., Nielsen, M. and Peters, B. (2012). Immune epitope database analysis resource. Nucleic Acids Research 40, 18

Klinman, N. and Aschinazi, G. (1971). The stimulation of splenic foci in vitro. Fournal of Immunology 106, 1338-1334.

Klysik, J. (2001). Concept of immunomics: a new frontier in the battle for gene function? Acta Biotheoretica 49, 191-202.

Kwong, P. D., Mascola, J. R. and Nabel, G. J. (2011). Rational design of vaccines to elicit broadly neutralizing antibodies to HIV-1. Cold Spring Harb Perspect Med 1, a007278.

Leca, M., Bornet, C., Montana, M., Curti, C. and Vanelle, P. (2015) Meningococcal vaccines: current state and future outlook. Pathologie Biologie 63, 144-151.

Loukas, A., Gaze, S., Mulvenna, J. P., Gasser, R. B., Brindley, P. J., Doolan, D. L., Bethony, J. M., Jones, M. K., Gobert, G. N., Driguez, P., McManus, D. P. and Hotez, P. J. (2011). Vaccinomics for the major blood feeding helminths of humans. Omics 15, 567-577.

Madura, F., Rizkallah, P. J., Holland, C. J., Fuller, A., Bulek, A., Godkin, A. J., Schauenburg, A. J., Cole, D. K. and Sewell, A. K. (2015). Structural basis for ineffective T-cell responses to MHC anchor residue-improved 'heteroclitic' peptides. European Fournal of Immunology 45, 584-591.

Margalit, H., Spouge, J. L., Cornette, J. L., Cease, K. B., Delisi, C. and Berzofsky, J. A. (1987). Prediction of immunodominant helper T cell antigenic sites from the primary sequence. Fournal of Immunology 138, 2213-2229.

McCarthy, J.S. and Good, M. F. (2010). Whole parasite blood stage malaria vaccines: a convergence of evidence. Human Vaccines 6, 114-123. McHeyzer-Williams, M., McLean, M., Lalor, P. and Nossal, G. (1993). Antigen-driven B cell differentiation in vivo. Fournal of Experimental Medicine 178, 295-307.

McWilliam, H. E. G., Driguez, P., Piedrafita, D., McManus, D. P. and Meeusen, E. N. T. (2012). Novel immunomic technologies for schistosome vaccine development. Parasite Immunology 34, 276-284.

Meeusen, E. N. T., Walker, J., Peters, A., Pastoret, P.-P. and Jungersen, G. (2007). Current status of veterinary vaccines. Clinical Microbiology Reviews 20, 489-510. 
Mehr, R. (2014). Immune system modeling and analysis. Frontiers in Immunology 5, 644 .

Mikolajczak, S. A., Lakshmanan, V., Fishbaugher, M., Camargo, N. Harupa, A., Kaushansky, A., Douglass, A. N., Baldwin, M., Healer, J., O'Neill, M., Phuong, T., Cowman, A. and Kappe, S. H. (2014). A next-generation genetically attenuated Plasmodium falciparum parasite created by triple gene deletion. Molecular Therapy 22, 17071715 .

Moise, L., Moss, S. F. and De Groot, A. S. (2012). Moving Helicobacter pylori vaccine development forward with bioinformatics and immunomics. Expert Review of Vaccines 11, 1031-1033.

Narang, V., Decraene, J., Wong, S. Y., Aiswarya, B. S., Wasem, A. R., Leong, S. R. and Gouaillard, A. (2012). Systems immunology: a survey of modeling formalisms, applications and simulation tools. Immunologic Research 53, 251-265.

Nielsen, M. and Lund, O. (2009). NN-align. An artificial neural network-based alignment algorithm for MHC class II peptide binding prediction. BMC Bioinformatics 10, 296

Osuna, C. E., Gonzalez, A.M., Chang, H.H., Hung, A. S., Ehlinger, E., Anasti, K., Alam, S. M. and Letvin, N. L. (2014). TCR affinity associated with functional differences between dominant and subdominant SIV epitope-specific CD8+ T cells in Mamu-A*01+ rhesus monkeys. PLoS Pathog 10, e1004069.

Ozen, S., Tucker, L. B. and Miller, L. C. (1998). Identification of Th subsets in juvenile rheumatoid arthritis confirmed by intracellular cytokine staining. Fournal of Rheumatology 25, 1651-1653.

Pizza, M., Scarlato, V., Masignani, V., Giuliani, M. M., Arico, B., Comanducci, M., Jennings, G. T., Baldi, L., Bartolini, E., Capecchi, B., Galeotti, C. L., Luzzi, E., Manetti, R., Marchetti, E., Mora, M., Nuti, S., Ratti, G., Santini, L., Savino, S., Scarselli, M., Storni, E., Zuo, P., Broeker, M., Hundt, E., Knapp, B., Blair, E., Mason, T., Tettelin, H., Hood, D. W., Jeffries, A. C. et al. (2000). Identification of vaccine candidates against serogroup B meningococcus by whole-genome sequencing. Science 287, 1816-1820.

Poland, G. A., Kennedy, R. B. and Ovsyannikova, I. G. (2011) Vaccinomics and personalized vaccinology: is science leading us toward a new path of directed vaccine development and discovery? PLoS Pathogens 7, e1002344.

Pouwels, K. B., Hak, E., van der Ende, A., Christensen, H., van den Dobbelsteen, G.P. and Postma, M. J. (2013). Cost-effectiveness of vaccination against meningococcal B among Dutch infants: crucial impact of changes in incidence. Human Vaccines $\&$ Immunotherapeutics 9, 1129-1138.

Pulendran, B. and Ahmed, R. (2006). Translating innate immunity into immunological memory: implications for vaccine development. Cell $\mathbf{1 2 4}$ 849-863.

Quintana, F. J., Hagedorn, P. H., Elizur, G., Merbl, Y., Domany, E. and Cohen, I. R. (2004). Functional immunomics: microarray analysis of IgG autoantibody repertoires predicts the future response of mice to induced diabetes. Proceedings of the National Academy of Sciences of the United States of America 2, 14615-14621.

Raman, K., Bhat, A. G. and Chandra, N. (2010). A systems perspective of host-pathogen interactions: predicting disease outcome in tuberculosis. Molecular BioSystems 6, 516-530.

Rammensee, H., Bachmann, J., Emmerich, N. P., Bachor, O. A. and Stevanovic, S. (1999). SYFPEITHI: database for MHC ligands and peptide motifs. Immunogenetics 50, 213-219.

Rappuoli, R. (2001). Reverse vaccinology, a genome-based approach to vaccine development. Vaccine 19, 2688-2691.

Rinaudo, C. D., Telford, J. L., Rappuoli, R. and Seib, K. L. (2009). Vaccinology in the genome era. The Fournal of Clinical Investigation 119, 2515-2525.

Sanchez-Valdez, F. J., Perez Brandan, C., Ferreira, A. and Basombrio, M. A. (2015). Gene-deleted live-attenuated trypanosoma cruzi parasites as vaccines to protect against Chagas disease. Expert Review Vaccines 14, 681-697.

Sanger, F., Nicklen, S. and Coulson, A. R. (1977). DNA sequencing with chain-terminating inhibitors. Proceedings of the National Academy of Sciences of the United States of America 74, 5463-5467.

Schellhammer, I. and Rarey, M. (2004). FlexX-Scan: fast, structurebased virtual screening. Proteins 57, 504-517.

Schetgen, M. (2014). Anti-pneumococcal vaccination in 2014. Revue Medicale de Bruxelles 35, 325-328.
Schubert, C. (2011). Systems immunology: complexity captured. Nature 473, 113-114

Schwartz, L., Brown, G. V., Genton, B. and Moorthy, V. S. (2012). A review of malaria vaccine clinical projects based on the WHO rainbow table. Malaria fournal 11, 1475-2875.

Serruto, D., Bottomley, M. J., Ram, S., Giuliani, M. M. and Rappuoli, R. (2012). The new multicomponent vaccine against meningococcal serogroup B, 4CMenB: immunological, functional and structural characterization of the antigens. Vaccine 30, 033.

Sette, A., Fleri, W., Peters, B., Sathiamurthy, M., Bui, H.-H. and Wilson, S. (2005). A roadmap for the immunomics of category A-C pathogens. Immunity 22, 155-161.

Sette, A. and Sundaram, R. (2006). The phenomenon of immunodomination: speculations on the nature of immunodominance. In Immunodominance - The Choice of the Immune System (ed. Frelinger, J. A.), pp. 57-72. Wyley-VCH Weinheim, Germany.

Sidney, J., Steen, A., Moore, C., Ngo, S., Chung, J., Peters, B. and Sette, A. (2010). Five HLA-DP molecules frequently expressed in the worldwide human population share a common HLA supertypic binding specificity. Fournal of Immunology 184, 2492-2503.

Siegrist, C.-A. (2013). 2 - Vaccine immunology. In Vaccines (Sixth Edition) (ed. Offit, S. A. P. A. O. A.), pp. 14-32. W.B. Saunders, London. Skinner, P. J., Daniels, M. A., Schmidt, C.S., Jameson, S. C. and Haase, A. T. (2000). Cutting edge: in situ tetramer staining of antigenspecific T cells in tissues. Fournal of Immunology 165, 613-617.

Slifka, M. K. and Amanna, I. (2014). How advances in immunology provide insight into improving vaccine efficacy. Vaccine 32, 2948-2957.

Sollner, J., Heinzel, A., Summer, G., Fechete, R., Stipkovits, L., Szathmary, S. and Mayer, B. (2010). Concept and application of a computational vaccinology workflow. Immunome Research 3, 1745-7580. Spurgeon, S. L., Jones, R. C. and Ramakrishnan, R. (2008). High throughput gene expression measurement with real time PCR in a microfluidic dynamic array. PLoS ONE 3, 0001662.

Stilling, R. M., Bordenstein, S. R., Dinan, T. G. and Cryan, J.F. (2014). Friends with social benefits: host-microbe interactions as a driver of brain evolution and development? Front Cell Infect Microbiol. 4, 147

Sun, P., Ju, H., Liu, Z., Ning, Q., Zhang, J., Zhao, X., Huang, Y., Ma, Z. and Li, Y. (2013). Bioinformatics resources and tools for conformational B-cell epitope prediction. Computational and Mathematical Methods in Medicine 2013, 11.

Tenzer, S., Peters, B., Bulik, S., Schoor, O., Lemmel, C., Schatz, M. M., Kloetzel, P.M., Rammensee, H. G., Schild, H. and Holzhutter, H. G. (2005). Modeling the MHC class I pathway by combining predictions of proteasomal cleavage, TAP transport and MHC class I binding. Cellular and Molecular Life Sciences 62, 1025-1037.

Tirani, M., Meregaglia, M. and Melegaro, A. (2015). Health and economic outcomes of introducing the New MenB Vaccine (Bexsero) into the Italian routine infant immunisation programme. PLOS ONE 10, $\mathrm{e} 0123383$.

Tournier, J.-N. and Quesnel-Hellmann, A. (2006). Host-pathogen interactions: a biological Rendez-Vous of the infectious nonself and danger models? PLoS Pathogens 2, e44.

Trieu, A., Kayala, M. A., Burk, C., Molina, D. M., Freilich, D. A., Richie, T. L., Baldi, P., Felgner, P.L. and Doolan, D. L. (2011) Sterile protective immunity to malaria is associated with a panel of novel P. falciparum antigens. Molecular \& Cellular Proteomics 10, 31

Vita, R., Overton, J. A., Greenbaum, J. A., Ponomarenko, J., Clark, J. D., Cantrell, J. R., Wheeler, D. K., Gabbard, J. L., Hix, D., Sette, A. and Peters, B. (2015). The immune epitope database (IEDB) $3 \cdot 0$. Nucleic Acids Research 43, 9.

Woodworth, J.S., Aagaard, C.S., Hansen, P. R., Cassidy, J.P., Agger, E. M. and Andersen, P. (2014). Protective CD4T cells targeting cryptic epitopes of Mycobacterium tuberculosis resist infection-driven terminal differentiation. Fournal of Immunology 192, 3247-3258.

Yao, B., Zheng, D., Liang, S. and Zhang, C. (2013). Conformational BCell epitope prediction on antigen protein structures: a review of current algorithms and comparison with common binding site prediction methods. PLoS ONE 8, e62249.

Zarnitsyna, V. I., Evavold, B. D., Schoettle, L. N., Blattman, J. N. and Antia, R. (2013). Estimating the diversity, completeness, and crossreactivity of the T cell repertoire. Frontiers in Immunology $\mathbf{4}, 485$. Zepp, F. (2010). Principles of vaccine design - lessons from nature. Vaccine 28, (Suppl. 3), C14-C24. 